\title{
Identical Particle Scattering From A Weakly Coupled Bose-Einstein Condensed Gas
}

\author{
A. Wynveen \\ A. Setty \\ A. Howard \\ J.W. Halley \\ C. E. Campbell
}

Follow this and additional works at: https://researchrepository.wvu.edu/faculty_publications

\section{Digital Commons Citation}

Wynveen, A.; Setty, A.; Howard, A.; Halley, J. W.; and Campbell, C. E., "Identical Particle Scattering From A Weakly Coupled BoseEinstein Condensed Gas" (2000). Faculty Scholarship. 557.

https://researchrepository.wvu.edu/faculty_publications/557 


\title{
Identical particle scattering from a weakly coupled Bose-Einstein condensed gas
}

\author{
A. Wynveen, ${ }^{1}$ A. Setty, ${ }^{2}$ A. Howard, ${ }^{3}$ J. W. Halley, ${ }^{1}$ and C. E. Campbell ${ }^{1}$ \\ ${ }^{1}$ School of Physics and Astronomy, University of Minnesota, Minneapolis, Minnesota 55455 \\ ${ }^{2}$ Department of Physics, West Virginia University, Morgantown, West Virginia 26506 \\ ${ }^{3}$ Department of Physics, Harvard University, Cambridge, Massachusetts 02138
}

(Received 28 January 2000; published 5 July 2000)

\begin{abstract}
We calculate the scattering states and cross sections for a Bose-Einstein condensed dilute gas trapped in a spherical square well of finite depth. The interactions are treated in the scattering length approximation. We solve the Gross-Pitaevskii equation and the Bogoliubov equations for bound and scattering states. The results show that there are transparency effects reminiscent of those conjectured to occur for strongly coupled systems. When incident particle wavelengths $\lambda$ are comparable to the well size $a$, exchange induced transparency enhancement is dramatic only for particular combinations of well depth, interaction strength, and particle number. For particles with large momenta $(a / \lambda \gg 1)$, however, exchange with the condensate results in enhanced transmission for all coupling strengths. We calculated the rate of decay of the scattering states to leading order in anharmonic corrections to the Bogoliubov approximation and found the corresponding inelastic cross sections to be extremely small.
\end{abstract}

PACS number(s): 03.75.Fi, 05.30.Jp, 67.40.Db

\section{INTRODUCTION}

In previous work we have suggested that finite size Bose systems in a Bose-Einstein condensed state may exhibit effective transparency to incident atoms indistinguishable from those that are condensed, as a consequence of the presence of the Bose condensation. Our suggestion was originally made for liquid helium [1] but, by similar qualitative arguments, a similar effect is to be expected for weakly coupled dilute Bose systems. Though variational calculations on strongly coupled helium have confirmed some aspects of our original suggestion [2], exact calculations of the scattering states of the strongly coupled system remain out of reach. For this reason, a calculation on a weakly coupled system, where the approximations are better controlled, is desirable. Further, the experimental creation of dilute Bose condensed systems of alkali-metal atoms [3-6] and spin-polarized hydrogen [7] gives rise to the possibility that an experiment like the one we proposed for liquid helium could be carried out on them.

For these reasons we have carried out a calculation [8] of the scattering states of a system of $N$ atoms in a finite spherical square well of radius $a$. The main qualitative feature that distinguishes this calculation from previous calculations [9-12] is the finite depth of the well, which is of course essential for a scattering problem for a system that is not self-bound. The cross sections that we find do exhibit a transparency effect, which, we will argue, arises from the same qualitative physical effects that led us to anticipate a transparency effect in liquid helium. When the wave vector $k$ of the incident particle is comparable to the inverse of the well radius $(k a \lesssim 1)$ we find that the transparency effects are dramatic only for certain combinations of model parameters due to effects of interference. However, for $k a \gg 1$ we always find enhanced transparency due to exchange of the incident particle with particles of the Bose condensed target. These features are qualitatively consistent with our earlier suggestions for the strongly interacting Bose-Einstein condensed system.

\section{MODEL AND CALCULATIONAL METHODS}

The calculation is carried out in the standard way [13] for a weakly coupled Bose system. The Hamiltonian is

$$
\begin{aligned}
H= & \int d \vec{r} \psi^{\dagger}(\vec{r})\left(\frac{-\hbar^{2} \nabla^{2}}{2 m}+V_{1}-\mu\right) \psi(\vec{r}) \\
& +\frac{1}{2} \iint d \vec{r} d \vec{r}^{\prime} \psi^{\dagger}(\vec{r}) \psi^{\dagger}\left(\vec{r}^{\prime}\right) V\left(\vec{r}, \vec{r}^{\prime}\right) \psi(\vec{r}) \psi\left(\vec{r}^{\prime}\right),
\end{aligned}
$$

where $\psi(\vec{r})$ is the wave operator for destruction of bosons at $\vec{r}, V_{1}$ is a confining potential, $\mu$ is the chemical potential, and $V\left(\vec{r}, \vec{r}^{\prime}\right)$ is the interaction between particles, for which we use the scattering length approximation [14]

$$
V\left(\vec{r}, \vec{r}^{\prime}\right)=\left(4 \pi \hbar^{2} a_{s} / m\right) \delta\left(\vec{r}-\vec{r}^{\prime}\right) .
$$

In the weak coupling limit [13] we proceed by writing $\psi(\vec{r})=\sqrt{N_{0}} \psi_{0}(\vec{r})+\delta \psi(\vec{r})$, where $\psi_{0}$ is a $c$ number which we take to be real and equal to the square root of the density of particles in the condensate at position $\vec{r}$. Minimizing the energy as a functional of $\psi_{0}(\vec{r})$ to zeroth order in the $\delta \psi(\vec{r})$ gives the Gross-Pitaevskii equation for $\psi_{0}$ :

$$
\left(\frac{-\hbar^{2} \nabla^{2}}{2 m}+V_{1}+N_{0} g\left|\psi_{0}(\vec{r})\right|^{2}\right) \psi_{0}(\vec{r})=\mu \psi_{0}(\vec{r}),
$$

where $g=4 \pi \hbar^{2} a_{s} / m$. The eigenvalue $\mu$ of this equation is the chemical potential. To first order in $\delta \psi(\vec{r})$ we obtain

$$
\begin{aligned}
i \hbar \frac{\partial \delta \psi}{\partial t}= & \left(\frac{-\hbar^{2} \nabla^{2}}{2 m}+V_{1}-\mu+2 g N_{0}\left|\psi_{0}(\vec{r})\right|^{2}\right) \delta \psi(\vec{r}) \\
& +N_{0} g\left|\psi_{0}(\vec{r})\right|^{2} \delta \psi^{\dagger}(\vec{r}) .
\end{aligned}
$$

Expanding 


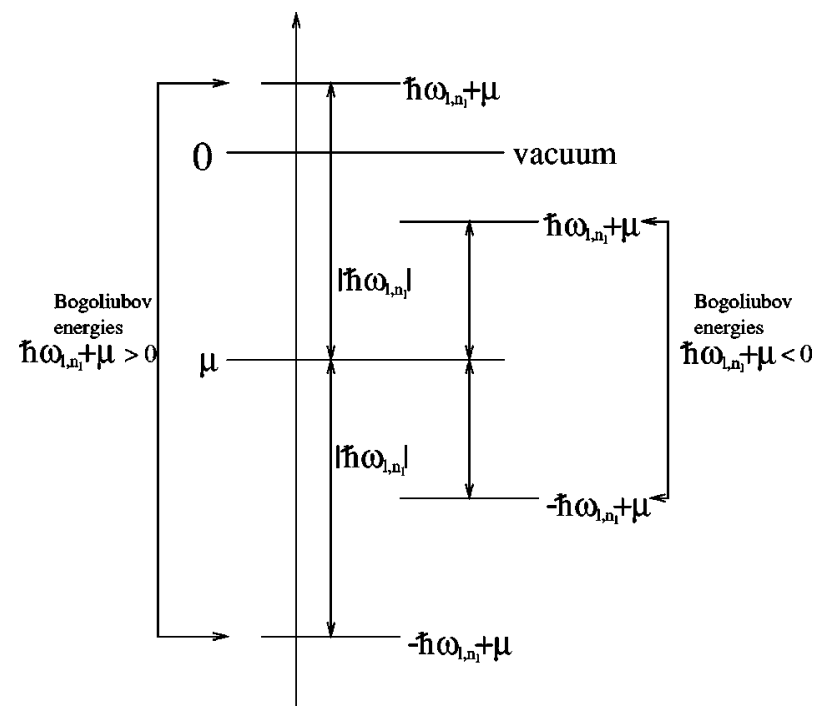

FIG. 1. Energies in continuum and bound solutions of the Bogoliubov equations. For Bogoliubov states for which $\hbar \omega_{l, n_{l}}+\mu$ $>0$, the allowed values of $\hbar \omega_{l, n_{l}}$ are continuous, and the $u_{l, n_{l}}$ components are unbound whereas the $v_{l, n_{l}}$ components are bound. When $\hbar \omega_{l, n_{l}}+\mu<0$, the allowed values of $\hbar \omega_{l, n_{l}}$ are discrete and both Bogoliubov components $u_{l, n_{l}}$ and $v_{l, n_{l}}$ are bound. The figure also shows the locations of $-\hbar \omega_{l, n_{l}}+\mu$, which may be regarded, for some purposes, as the energy of the $v$ component, in the continuum and discrete cases.

$$
\delta \psi(\vec{r}, t)=\sum_{\nu}\left[U_{\nu}(\vec{r}) b_{\nu} e^{-i \omega_{\nu} t}+V_{\nu}(\vec{r}) b_{\nu}^{\dagger} e^{i \omega_{\nu} t}\right]
$$

gives the Bogoliubov equations for the excited states:

$\left(-\frac{\hbar^{2}}{2 m} \nabla^{2}+V_{1}-\mu+2 N_{0} g \psi_{0}^{2}-\hbar \omega_{\nu}\right) U_{\nu}(\vec{r})+N_{0} g \psi_{0}^{2} V_{\nu}=0$

$\left(-\frac{\hbar^{2}}{2 m} \nabla^{2}+V_{1}-\mu+2 N_{0} g \psi_{0}^{2}+\hbar \omega_{\nu}\right) V_{\nu}(\vec{r})+N_{0} g \psi_{0}^{2} U_{\nu}=0$,

where $\nu$ contains the quantum numbers specifying the excited states (three of them in three dimensions). $\hbar \omega_{\nu}$ is the energy of the excited state measured relative to $\mu$. The energy of the excited state measured relative to the vacuum zero is $\hbar \omega_{\nu}+\mu$.

The boundary conditions for these equations are as follows. For the Gross-Pitaevskii equation we require that $\psi_{0}(r) \rightarrow$ const $\times \exp \left(-\sqrt{2|\mu| m / \hbar^{2}} r\right) / r$ for large $r$ and that $\psi_{0} \rightarrow$ const at the origin. We consider only real, spherically symmetric solutions of the Gross-Pitaevskii equation here. For the Bogoliubov equations the boundary conditions depend on whether $\hbar \omega_{\nu}+\mu>0$ or $\hbar \omega_{\nu}+\mu<0$, corresponding to the continuum and the bound states, respectively. Because the existence of a continuum is a relatively unfamiliar feature of this calculation we provide a sketch in Fig. 1. We confine attention to spherically symmetric $V_{1}$ and define functions $u_{l, n_{l}}(r)$ and $v_{l, n_{l}}(r)$ by the relations

$$
\begin{aligned}
& U_{\nu}(\vec{r})=Y_{l}^{m}(\vec{r}) u_{l, n_{l}}(r) / r, \\
& V_{\nu}(\vec{r})=Y_{l}^{m}(\vec{r}) v_{l, n_{l}}(r) / r .
\end{aligned}
$$

Then the Bogoliubov equations become

$$
\begin{gathered}
\frac{d^{2} u_{l, n_{l}}}{d \rho^{2}}-\left(v_{1}+l(l+1) / \rho^{2}+16 \pi N_{0} a_{s} a^{2} \psi_{0}^{2}\right. \\
\left.-\frac{\hbar \omega_{l, n_{l}}+\mu}{\hbar \omega_{0}}\right) u_{l, n_{l}}-8 \pi N_{0} a_{s} a^{2} \psi_{0}^{2} v_{l, n_{l}}=0, \\
\frac{d^{2} v_{l, n_{l}}}{d \rho^{2}}-\left(v_{1}+l(l+1) / \rho^{2}+16 \pi N_{0} a_{s} a^{2} \psi_{0}^{2}\right. \\
\left.-\frac{-\hbar \omega_{l, n_{l}}+\mu}{\hbar \omega_{0}}\right) v_{l, n_{l}}-8 \pi N_{0} a_{s} a^{2} \psi_{0}^{2} u_{l, n_{l}}=0 .
\end{gathered}
$$

We have expressed the equations in a dimensionless form by dividing through by $\hbar \omega_{0}=\hbar^{2} / 2 m a$, where $a$ is the well radius, $\rho=r / a$, and $v_{1}=V_{1} / \hbar \omega_{0} . n_{l}$ labels solutions to these equations with different numbers of nodes in the case of bound states. In the case of the continuum, $n_{l}$ is replaced by the energy. The boundary conditions are, if $\hbar \omega_{l, n_{l}}>|\mu|$ (continuum),

$$
u_{l, n_{l}} \rightarrow r j_{l}\left[k r+\delta_{l}(k)\right] \text { as } r \rightarrow \infty
$$

$$
v_{l, n_{l}}(r) \rightarrow \text { const } \times e^{-\kappa r} \text { as } r \rightarrow \infty
$$

where

$$
k=\sqrt{2 m\left(\hbar \omega_{l, n_{l}}+\mu\right) / \hbar^{2}}
$$

and

$$
\kappa=\sqrt{2 m\left(\hbar \omega_{l, n_{l}}-\mu\right) / \hbar^{2}}
$$

On the other hand, if $\hbar \omega_{l, n_{l}}<|\mu|$ (bound states),

$$
\begin{aligned}
& u_{l, n_{l}}(r) \rightarrow \text { const } \times e^{-\kappa^{\prime} r} \text { as } r \rightarrow \infty, \\
& v_{l, n_{l}}(r) \rightarrow \operatorname{const} \times e^{-\kappa r} \text { as } r \rightarrow \infty,
\end{aligned}
$$

in which $\kappa^{\prime}=\sqrt{2 m\left(-\hbar \omega_{l, n_{l}}-\mu\right) / \hbar^{2}}$. At the origin the boundary condition is $u_{l, n_{l}}, v_{l, n_{l}} \propto r^{l+1}$. Notice that the $v$ component of the wave function is always bound, even in the continuum. This makes excellent physical sense, because the $v$ component is to be interpreted as the amplitude for adding a particle to the condensate and this can only be accomplished physically in the region where the system is dense. 


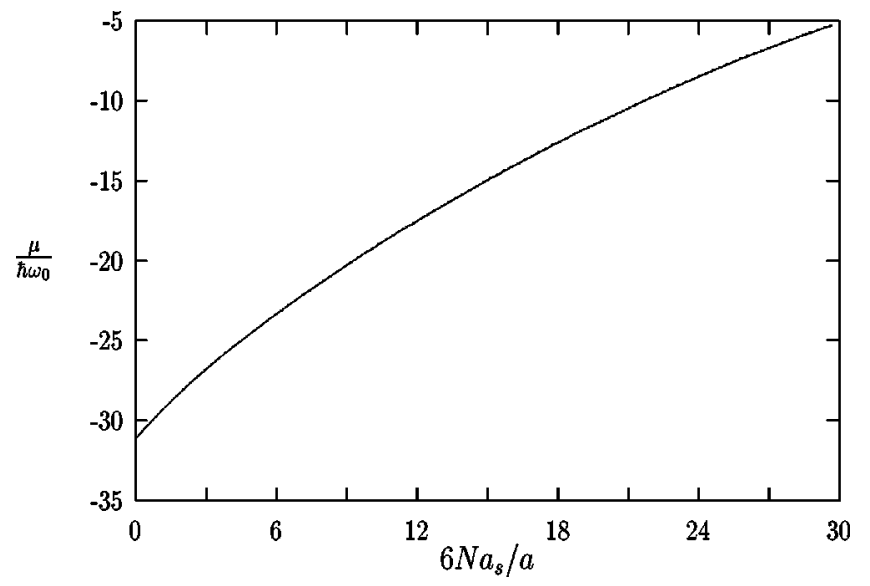

FIG. 2. Chemical potential as a function of scattering length. The depth of the well is $38.44 \hbar \omega_{0}$ and $N_{0}=1000$.

We solve these equations numerically by integrating out from the origin with specific initial conditions to achieve the required behavior at large $r$. In the case of bound states (which are not the primary focus of the present paper), the energy is adjusted along with the initial conditions of the $u$ and $v$ components of the wave function such that these components simultaneously approach zero for large $r$. Experiments on Bose condensed alkali-metal vapors have confirmed $[10,15,16]$ that the predicted bound states are quite close to the observed ones. For continuum states, the initial conditions of the wave function components are varied such that the $v$ component converges to zero. The phase shift is determined by fitting the $u$ component's known form at large $r$. (We smooth the potential $V_{1}$ at the step by a small amount for numerical reasons, replacing the step by a Fermi function of a width that is larger than the step size but smaller than the scale of the variation of the wave function. For our parametrization this turns out to be easy to achieve.) The results are expressed in dimensionless form, measuring energy in units of $\hbar \omega_{0}$ and lengths in units of $a$. (For alkali-metal experiments, characteristic values of $a$ are of the order of $10^{4} \AA$. With this value, the range of $a_{s} / a$ chosen here spans realistic values for alkali metals.) In interpreting the magnitudes of the scattering lengths in these units, it is helpful to note that the mean field energy per particle arising from the condensate in this model is approximately $N g / \Omega$ where $\Omega$ is the size (volume) of the condensate wave function and $N$ is the number of particles in the system (neglecting depletion in this rough approximation). In units of $\hbar \omega_{0}$ (characteristic of single particle kinetic energies in the spherical well) this is $N g / \Omega=6 N\left(a_{s} / a\right) \hbar \omega_{0}$.

\section{RESULTS}

We present results for $N=1000$ and a well depth of $38.44 \hbar \omega_{0}$. Since the mean field energy per particle can be written as $6 N\left(a_{s} / a\right) \hbar \omega_{0}$, the scattering lengths are presented in the dimensionless form $6 \mathrm{Na} / \mathrm{a}$ with a range 0 $<6 N a_{s} / a<32$ such that mean field energies approaching

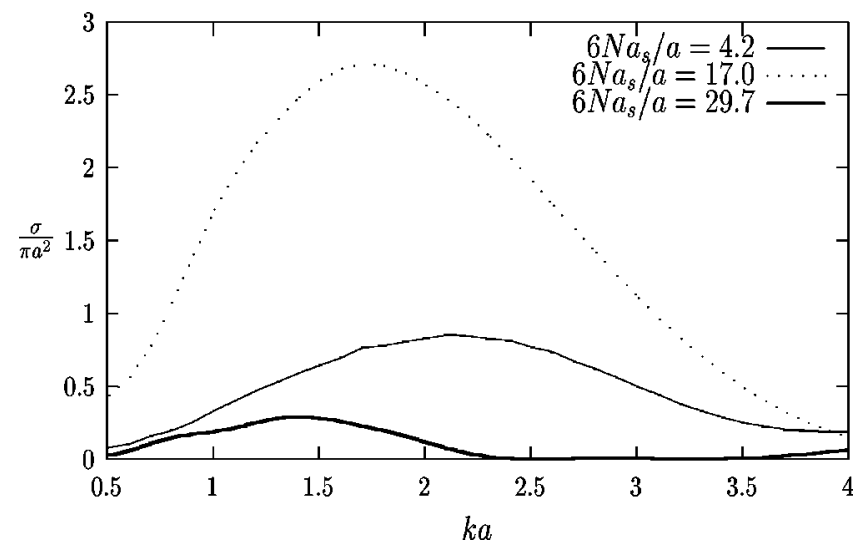

FIG. 3. Cross sections for a series of scattering lengths. The well width is $a$ and the well depth is $38.44 \hbar \omega_{0}$.

the well depth are probed. Values of the chemical potential as a function of coupling strength are shown in Fig. 2. The chemical potential is approaching zero as the coupling strength approaches the end of the range considered. This is because the repulsive interactions are making such a large mean field that the particles could not be held in so shallow a trap if the coupling were to be increased much more.

Turning to excited states, we exhibit cross sections, calculated from

$$
\sigma_{l}(k)=4 \pi(2 l+1) \sin ^{2} \delta_{l}(k) / k^{2}
$$

for the $l=1$ channel in Figs. 3 and 4. For comparison, we show the cross section calculated by use of the modified equation in which the term involving $v_{l, n_{l}}$ has been set to zero so that the scattering problem is just the scattering of a particle from the Hartree-like mean field due to the Gross Pitaevskii condensate (Fig. 5). The full solutions, by contrast, include the $v_{l, n}$, which corresponds physically to in-

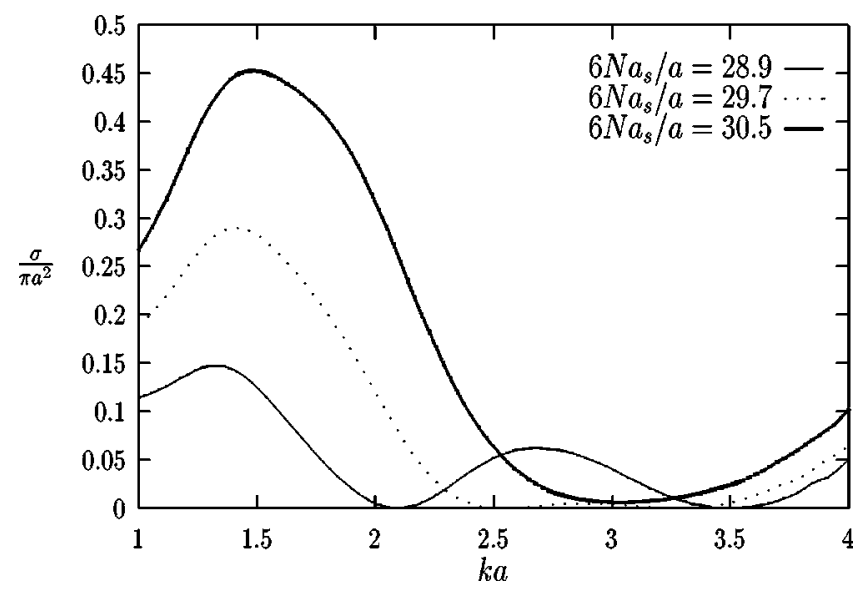

FIG. 4. Cross sections near transparency. The well width is $a$ and the well depth is $38.44 \hbar \omega_{0}$. 


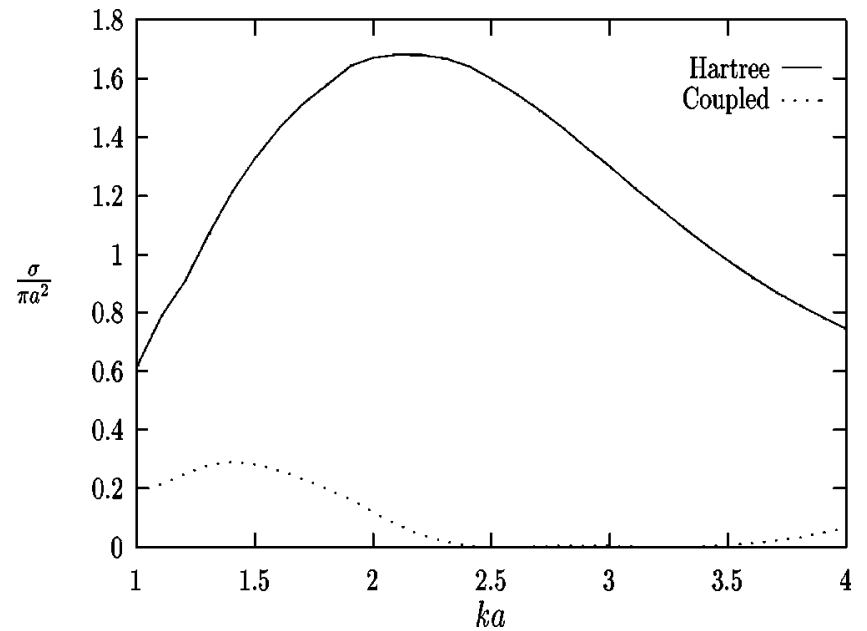

FIG. 5. Hartree and condensate coupled cross sections. Both cross sections given for a scattering length of $6 \mathrm{Na} a_{s} / a=29.7$.

cluding the possibility that the scattered particle is exchanged with the condensate during the scattering process. One finds that, at small coupling strengths, the scattering cross section is almost entirely taken into account by theHartree-like mean field effect, but that, as the scattering length increases, the exchange effects are increasingly large (but these effects do not change monotonically with the coupling strength). At $6 N a_{s} / a=30$ we see a huge effect, in which the cross section almost disappears in the $l=1$ channel, corresponding to nearly complete transparency. However, for still larger couplings, the system becomes increasingly opaque again. We can describe the conditions required for this transparency to occur in terms of the dimensionless quantities $N g\left|\psi_{0}(0)\right|^{2} a^{2}$ [where $\left|\psi_{0}(0)\right|$ corresponds to the amplitude of the Gross-Pitaevskii ground-state wave function at $\vec{r}=0]$ and $v_{1}$ as shown in Fig. 6 .

In the calculations reported above, in which $k a$ was of order unity, we observed exchange enhanced transparency for specific coupling strengths. However, for other couplings in the range of momenta shown in Figs. 3 and 4, we find that

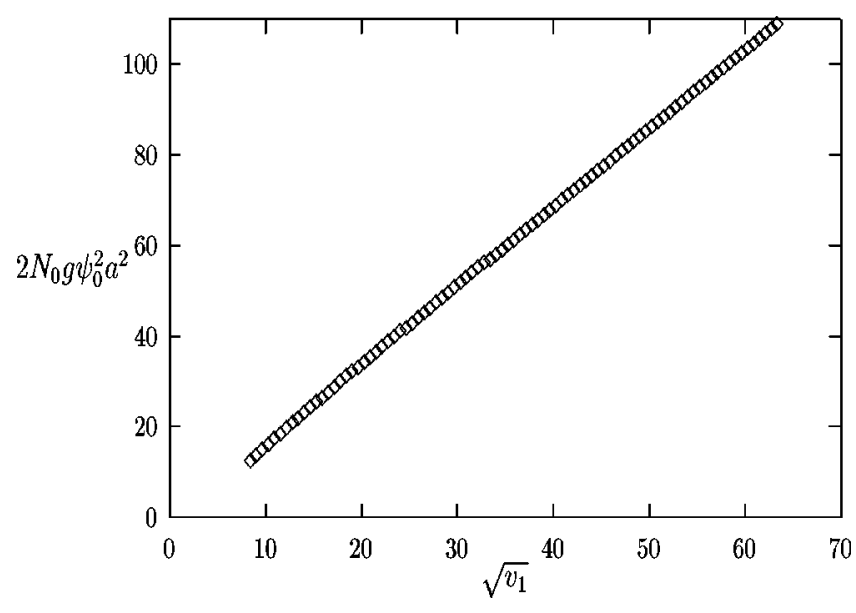

FIG. 6. Location of transparency for range of dimensionless parameters. The value for $\psi_{0}^{2}$ is taken where $r=0$.

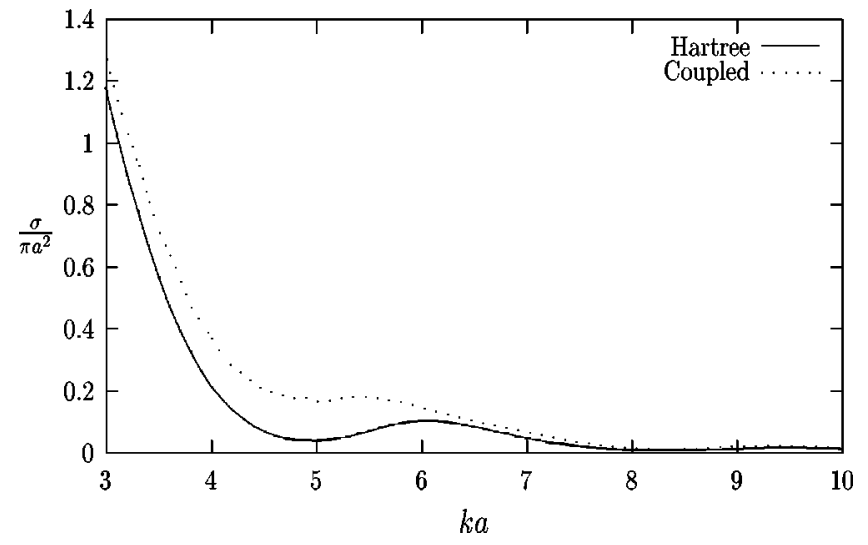

FIG. 7. Hartree and condensate coupled cross sections for a scattering length corresponding to $6 N a_{s} / a=12.7$. For this incident particle momentum range the Hartree cross section is on average smaller than the condensate coupled cross section.

the effects of exchange can result in cross sections larger than those for the Hartree case. We attribute this nonmonotonicity in the effect of exchange on the cross section to effects in which the internally reflected waves interfere with each other in the target during the scattering process. On the other hand, when $k a \gg 1$, the $l=1$ cross sections that we find when exchange is included in the calculation are always smaller than those where there is no exchange. This is presumably because, at these larger momenta, interference effects are reduced since the incident particle wave vector is much larger than the size of the system. Figures 7 and 8 show the condensate coupled and Hartree cross sections for a scattering length corresponding to $6 \mathrm{Na} / \mathrm{a}=12.7$. Here the condensate coupled cross section is larger than that for the Hartree case for incident particles when $k a \sim 1$. However, for incident particles of larger momentum, the condensate coupled cross section is smaller. We find that similarly for all coupling strengths the exchange reduces the cross section at large momentum: For large $k a$ and $l=1$ we show the ratio of the average condensate coupled cross section to the Hartree-

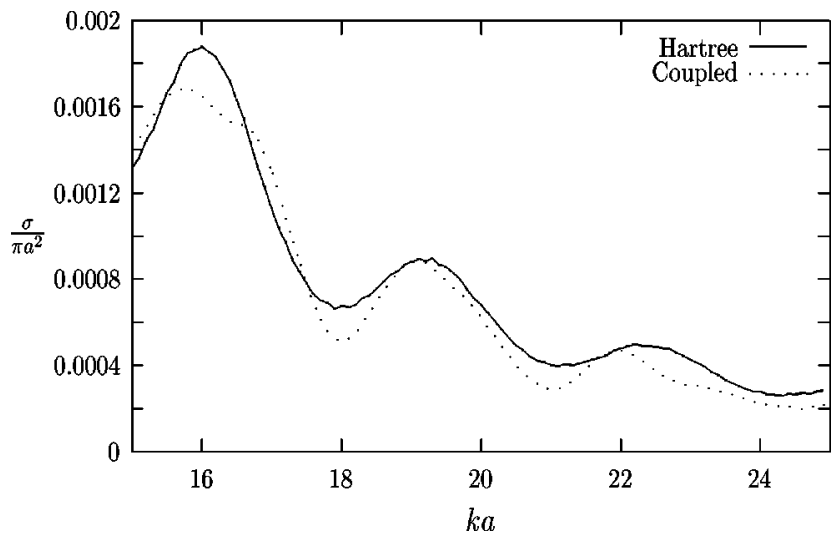

FIG. 8. Hartree and condensate coupled cross sections for a scattering length corresponding to $6 N a_{s} / a=12.7$. For this incident particle momentum range where $k a \gg 1$, the condensate coupled cross section is on average smaller than the Hartree cross section. 


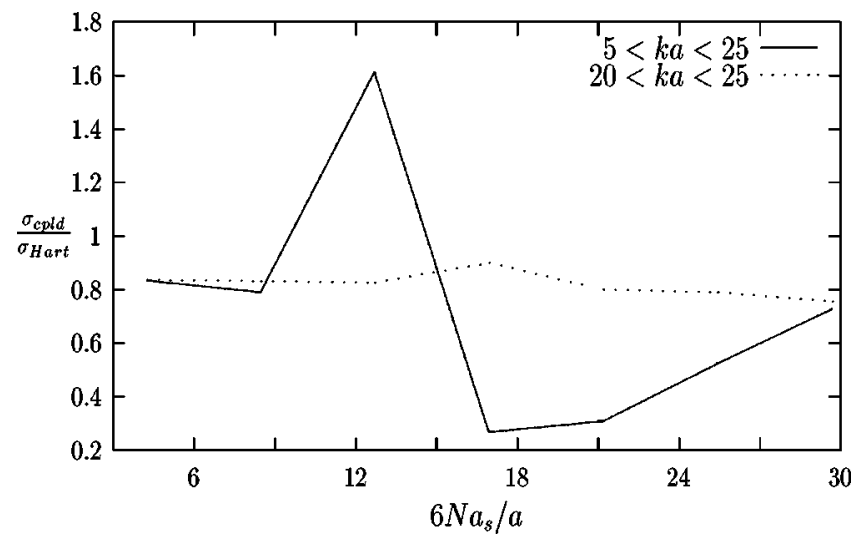

FIG. 9. Ratio of condensate coupled to Hartree cross sections. The solid line corresponds to the ratio of the average cross sections over a momentum range of $5<k a<25$ for a series of coupling strengths. The dotted line corresponds to the ratio of cross sections over a momentum range of $20<k a<25$, which is always smaller than 1 . This suggests that transmission, for all couplings, of larger momentum incident particles is enhanced when there is exchange.

cross section for two different momentum ranges as a function of scattering length in Fig. 9. The transmission is exchange enhanced for all couplings for incident particles with momenta $k a \gg 1$.

As expected on physical grounds, the largest transparency effect was seen in the $l=1$ channel. However, we found that other contributing angular momentum channels also showed the same effect, as shown in Figs. 10 and 11. As with the $l$ $=1$ channel, we again observe exchange induced enhancement for $k a \gg 1$ in these other channels (up to $l=3$ ) over the same range of coupling strengths. As an example, Fig. 10 shows the Hartree and condensate coupled cross sections at large incident particle momenta for $l=2$ at the coupling that yielded transparency in the $l=1$ cross section.

We also determined the total cross section for the momenta, $k a \sim 1$, and coupling strength where transparency was observed in the $l=1$ channel (Figs. 3 and 4). Summing the relevant partial cross sections up to $l=4$ (larger values of $l$

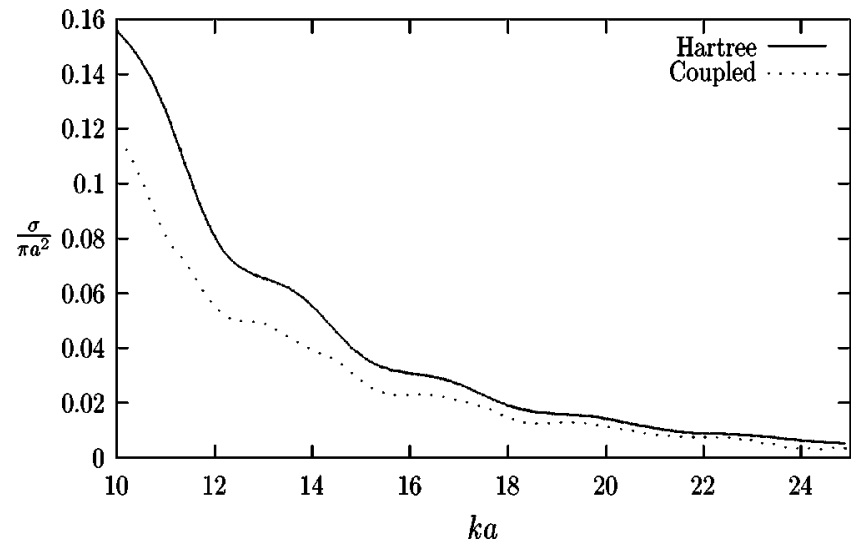

FIG. 10. Hartree and condensate coupled cross sections for $l$ $=2$ and a scattering length of $6 N a_{s} / a=29.7$. As with the $l=1$ cross section, we again see a reduced cross section due to exchange for other momentum channels.

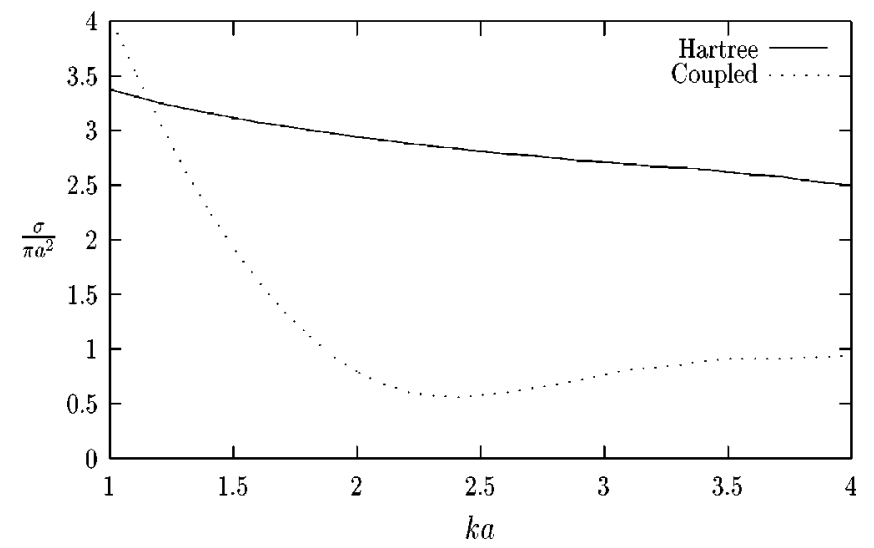

FIG. 11. Hartree and condensate coupled total cross sections at the coupling strength where transparency is seen for $k a \sim 1$. The total cross section also displays an exchange induced enhanced transmission like that for the $l=1$ partial cross section.

do not significantly contribute to the total cross section for this range of momentum), we find the total cross section shown in Fig. 11. The total cross section including exchange is seen to be smaller than that for the Hartree case over much of this momentum range and exhibits a pronounced dip where the large transparency occurs in the $l=1$ channel.

In Fig. 12 we show the $u$ and $v$ components of the wave function of the scattering state corresonding to a scattering length that gives a large transparency effect in the $l=1$ scattering channel. $v$ is seen to be large and nodeless, corresponding to a perturbation of the spherical condensate very nearly like a uniform translation. This is consistent with our earlier suggestion that the incident atom would mix virtually with a state corresponding to a boosted $(N+1)$-particle ground state, resulting in transparency.

These calculations were carried out assuming that $N$ $=N_{0}$ in the Bogoliubov equations, neglecting depletion. To check this assumption, we calculated the depletion of the condensate due to the interactions by use of the well known relation

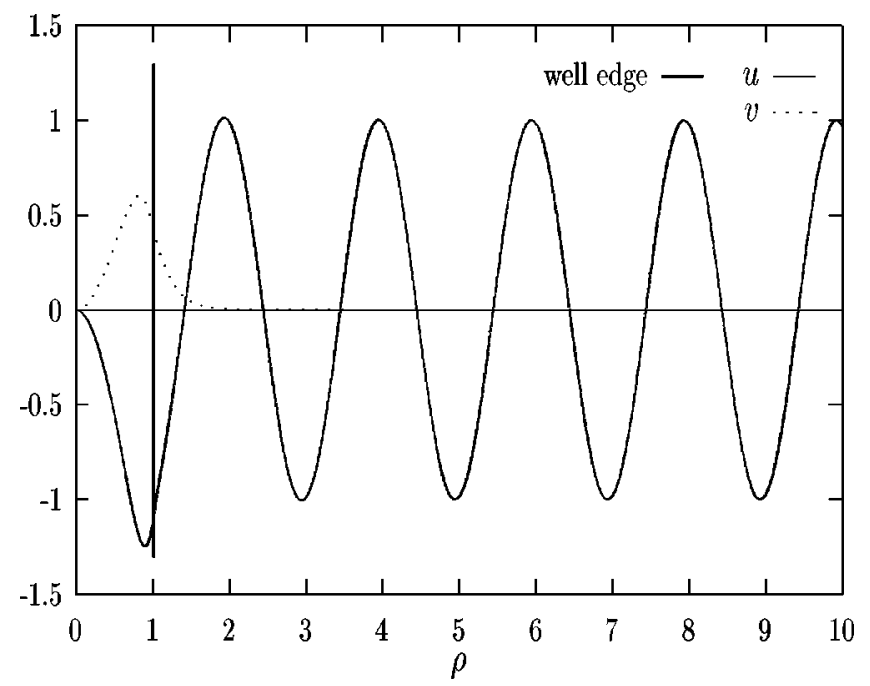

FIG. 12. $u$ and $v$ components of the wave function. Components are shown corresponding to $k a=3.1$ and $6 N a_{s} / a=29.7$ where the tranparency is seen. 


$$
N-N_{0}=\int d \vec{r}\left\langle\delta \psi^{\dagger}(\vec{r}) \delta \psi(\vec{r})\right\rangle=\sum_{\nu}\left|V_{\nu}\right|^{2} .
$$

We found that the bound states contribute more to the depletion than those in the continuum, but that for all coupling strengths the depletion of the condensate was insignificant. For example, the condensate fraction $N_{0} / N$ for a scattering length of $6 \mathrm{Na} / \mathrm{a}=30$ was approximately 0.995 and varied from this value by only about \pm 0.002 over the entire range of scattering lengths considered here. Thus, self-consistent calculations that took account of depletion would not significantly change the results reported here.

Several workers have suggested that the proposed transparency effect in superfluid helium-4 [1,2] would be masked by inelastic scattering processes. In the context of the weakly interacting model studied in the present paper, we can calculate the effects of inelasticity in a controlled manner. To do this, we considered the effect of higher order terms in the deviation operators $\delta \psi$ and $\delta \psi^{\dagger}$ in the Hamiltonian Eq. (1). The rate of decay of the scattering states, up to third order of the deviation operators, is given by

$$
R_{i \rightarrow f}=\sum_{\text {final }} \frac{2 \pi}{\hbar}\left|\left\langle i\left|\hat{H}_{3}\right| f\right\rangle\right|^{2} \delta\left(E_{i}-E_{f}\right)
$$

where

$$
\hat{H}_{3}=\sqrt{N_{0}} g \int d \vec{r} \delta \psi^{\dagger}(\vec{r}) \psi_{0}^{*}(\vec{r}) \delta \psi(\vec{r}) \delta \psi(\vec{r})+\text { H.c. }
$$

Using the wave function components found in Eq. (8), we were able to compute (see the Appendix) the rate of decay for the $l=1$ scattering state. The inelastic cross section can then be easily determined by dividing the transition probability rate by the flux of the incident particle,

$$
\sigma_{i n}=\frac{R_{i \rightarrow f}}{\hbar k / m} .
$$

Figure 13 shows the total inelastic cross section for the $l$ $=1$ scattering state in the range of interest. As can be seen from this graph, the inelastic cross section is about five orders of magnitude smaller than the corresponding elastic cross section at the scattering length that yields the transparency in the $l=1$ channel. This demonstrates that inelastic effects play a very minor role in this scattering process and have little effect on the transparency seen in the elastic cross section.

\section{DISCUSSION AND CONCLUSIONS}

In this paper, we report results of a calculation of the scattering of a Bose particle from a collection of identical Bose particles interacting weakly repulsively in scattering length approximation at zero temperature and confined by a spherical well of finite depth. We find that the exchange effects in the Bogoliubov equations, which arise from presence of the Bose condensate through processes in which the

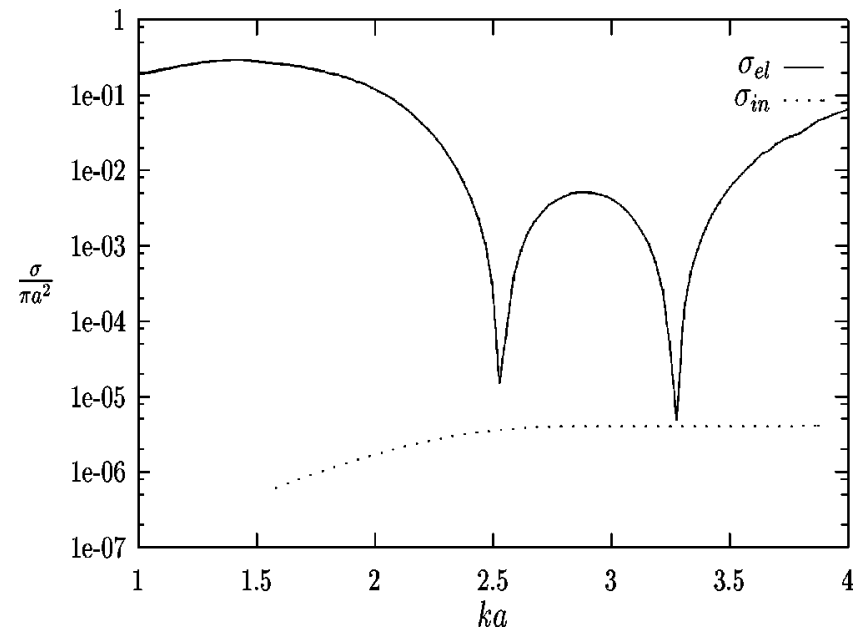

FIG. 13. Elastic and inelastic cross sections. Cross sections for a coupling of $6 \mathrm{Na} \mathrm{a}_{s} / \mathrm{a}=29.7$ where transparency is observed.

wave function of the incident particle mixes quantum mechanically with states in the condensate, give rise to a reduced cross section (enhanced transparency) relative to the case in which such exchange effects are absent, as long as the wave vector $k$ of the incident particle satisfies $k a \gg 1$. For $k a \lesssim 1$ we also find large effects due to exchange, but, presumably because of the effects of interference of the scattered wave with itself, the effect of exchange can have either sign (enhanced or reduced transparency) depending on the values of $k$, the scattering length, and the depth and width of the confining potential. For a Gaussian potential well, we found little or no exchange induced transparency effect in this $k a \lesssim 1$ range.

By going beyond the Bogoliubov approximation, we calculated the effects of anharmonicity leading to dissipation in the present model and found that the inelastic cross section was completely negligible in the present context. This means that exchange enhanced transparency would not be masked by dissipation in a weakly interacting system of this type.

We believe that these results are qualitatively consistent with the ideas and calculations published earlier on strongly coupled Bose systems, [1,2] in which we suggested that exchange enhanced transparency might be observed in such systems. This consistency does not, of course, constitute a proof that those ideas and calculations correctly predict the expected transparency in strongly coupled systems, because many approximations and assumptions were required to make the earlier calculations. Nevertheless, the present results are encouraging to the strongly interacting program, because it is possible to imagine that the weak coupling calculations might have been inconsistent with the qualitative results in the work on strongly coupled systems and they are not.

It appears to us that it might be possible to carry out experiments of the sort modeled here on Bose-Einstein condensed alkali-metal or hydrogen systems. Confinement in Gaussian wells is well known and some control of the well shape appears to be possible. The main unexplored aspect is the production of a beam. Confinement of two clouds of 
Bose-Einstein condensed alkali-metal gas has been achieved and interference effects have been observed [17]. A version of such an experiment in which one of the clouds was Bose condensed and the other was more dilute and confined to a smaller space before being released to impact the larger, Bose condensed system would begin to approximate the conditions modeled in the calculation reported here.

\section{ACKNOWLEDGMENTS}

This work was supported in part by a Graduate Student Research Project Grant from NASA, by the Minnesota Supercomputing Institute, by NSF REU Grant No. NSF/CDA9527932-002, by the University of Minnesota Graduate School and Department of Physics, by a grant from Shell Oil
Company to the University of Minnesota Physics Department, and by the National Science Foundation.

\section{APPENDIX: CALCULATION OF THE RATE OF DECAY OF THE BOGOLIUBOV SCATTERING STATES}

To calculate the transition probability rate of decay, we first expand the deviation operators in the perturbing Hamiltonian Eq. (14), using Eq. (5). The components of the Bogoliubov expansion can in turn be written, again, as a product of spherical harmonic and radial functions [Eq. (7)] due to the spherical symmetry invoked in the calculation.

Because of the form of the perturbing Hamiltonian, only certain combinations of these components contribute to the decay rate. After integrating over the solid angle, the transition probability rate of the $l=1$ scattering state reduces to

$$
\begin{aligned}
& R_{i \rightarrow f}=\frac{32 N_{0} \hbar a_{s}^{2}}{\pi m} \sum_{l^{\prime}, m^{\prime}, m} \int \frac{d q^{\prime}}{\sqrt{k^{2}-q^{\prime 2}}}\left(\sqrt{\frac{\left(2 l^{\prime}+1\right)\left[2\left(l^{\prime} \pm 1\right)+1\right]}{4 \pi(3)}}\left\langle l^{\prime}\left(l^{\prime} \pm 1\right) ; m^{\prime}\left(m-m^{\prime}\right) \mid l^{\prime}\left(l^{\prime} \pm 1\right) ; 1 m\right\rangle\right. \\
& \times\left\langle l^{\prime}\left(l^{\prime} \pm 1\right) ; 00 \mid l^{\prime}\left(l^{\prime} \pm 1\right) ; 10\right\rangle \int \frac{d r}{r} \psi_{0} u_{k, 1} u_{q^{\prime}, l^{\prime}} u_{\left(\sqrt{2 m / \hbar^{2}} \sqrt{\left.\epsilon_{k}-\epsilon_{q^{\prime}}\right)} l^{\prime} \pm 1\right.}+\sqrt{\frac{(3)\left[2\left(l^{\prime} \pm 1\right)+1\right]}{4 \pi\left(2 l^{\prime}+1\right)}} \\
& \times\left\langle 1\left(l^{\prime} \pm 1\right) ; m\left(m^{\prime}-m\right) \mid 1\left(l^{\prime} \pm 1\right) ; l^{\prime} m^{\prime}\right\rangle\left\langle 1\left(l^{\prime} \pm 1\right) ; 00 \mid 1\left(l^{\prime} \pm 1\right) ; l^{\prime} 0\right\rangle \int \frac{d r}{r} \psi_{0} v_{k, 1} v_{q^{\prime}, l^{\prime}} u_{\left(\sqrt{2 m / \hbar^{2}} \sqrt{\epsilon_{k}-\epsilon_{q^{\prime}}} l^{\prime} \pm 1\right.} \\
& +\sqrt{\frac{(3)\left(2 l^{\prime}+1\right)}{4 \pi\left[2\left(l^{\prime} \pm 1\right)+1\right]}}\left\langle 1 l^{\prime} ; m m^{\prime} \mid 1 l^{\prime} ;\left(l^{\prime} \pm 1\right)\left(m+m^{\prime}\right)\right\rangle\left\langle 1 l^{\prime} ; 00 \mid 1 l^{\prime} ;\left(l^{\prime} \pm 1\right) 0\right\rangle \\
& \times \int \frac{d r}{r} \psi_{0} v_{k, 1} u_{q^{\prime}, l^{\prime}} v_{\left(\sqrt{2 m / \hbar^{2}} \sqrt{\left.\epsilon_{k}-\epsilon_{q^{\prime}}\right)} l^{\prime} \pm 1\right.}+\sqrt{\frac{(3)\left(2 l^{\prime}+1\right)}{4 \pi\left[2\left(l^{\prime} \pm 1\right)+1\right]}}\left\langle 1 l^{\prime} ; m m^{\prime} \mid 1 l^{\prime} ;\left(l^{\prime} \pm 1\right)\left(m+m^{\prime}\right)\right\rangle \\
& \times\left\langle 1 l^{\prime} ; 00 \mid 1 l^{\prime} ;\left(l^{\prime} \pm 1\right) 0\right\rangle \int \frac{d r}{r} \psi_{0} u_{k, 1} v_{q^{\prime}, l^{\prime}} u_{\left(\sqrt{2 m / \hbar^{2}} \sqrt{\epsilon_{k}-\epsilon_{q^{\prime}}}\right) l^{\prime} \pm 1}+\sqrt{\frac{(3)\left[2\left(l^{\prime} \pm 1\right)+1\right]}{4 \pi\left(2 l^{\prime}+1\right)}} \\
& \times\left\langle 1\left(l^{\prime} \pm 1\right) ; m\left(m^{\prime}-m\right) \mid 1\left(l^{\prime} \pm 1\right) ; l^{\prime} m^{\prime}\right\rangle\left\langle 1\left(l^{\prime} \pm 1\right) ; 00 \mid 1\left(l^{\prime} \pm 1\right) ; l^{\prime} 0\right\rangle \int \frac{d r}{r} \psi_{0} u_{k, 1} u_{q^{\prime}, l^{\prime}} v_{\left(\sqrt{2 m / \hbar^{2}} \sqrt{\epsilon_{k}-\epsilon_{q^{\prime}}} l^{\prime} \pm 1\right.} \\
& +\sqrt{\frac{\left(2 l^{\prime}+1\right)\left[2\left(l^{\prime} \pm 1\right)+1\right]}{4 \pi(3)}}\left\langle l^{\prime}\left(l^{\prime} \pm 1\right) ; m^{\prime}\left(m-m^{\prime}\right) \mid l^{\prime}\left(l^{\prime} \pm 1\right) ; 1 m\right\rangle\left\langle l^{\prime}\left(l^{\prime} \pm 1\right) ; 00 \mid l^{\prime}\left(l^{\prime} \pm 1\right) ; 10\right\rangle \\
& \left.\times \int \frac{d r}{r} \psi_{0} v_{k, 1} v_{q^{\prime}, l^{\prime}} v_{\left(\sqrt{2 m / \hbar^{2}} \sqrt{\epsilon_{k}-\epsilon_{q^{\prime}}}\right) l^{\prime} \pm 1}\right)^{2},
\end{aligned}
$$

where the $\langle\cdots \mid \cdots\rangle$ terms are Clebsch-Gordan coefficients. The integrals on $r$ were evaluated numerically using the wave functions $u$ and $v$ which were determined from Eq. (8) as described in the text. The numerical calculation revealed that the contribution to the decay rate due to larger $l^{\prime}$ values diminished quickly such that the sum on $l^{\prime}$ could be truncated at a value of 4 . 
[1] J.W. Halley, C.E. Campbell, C.F. Giese, and K. Goetz, Phys. Rev. Lett. 71, 2429 (1993).

[2] A. Setty, J.W. Halley, and C.E. Campbell, Phys. Rev. Lett. 79, 3930 (1997).

[3] M.H. Anderson, J.R. Ensher, M.R. Matthews, C.E. Wieman, and E.A. Cornell, Science 269, 198 (1995).

[4] C.C. Bradley, C.A. Sackett, J.J. Tollett, and R.G. Hulet, Phys. Rev. Lett. 75, 1687 (1995).

[5] C.C. Bradley, C.A. Sackett, and R.G. Hulet, Phys. Rev. Lett. 78, 985 (1997).

[6] K.B. Davis, M.-O. Mewes, M.R. Andrews, N.J. van Druten, D.S. Durfee, D.M. Kurn, and W. Ketterle, Phys. Rev. Lett. 75, 3969 (1995).

[7] D.G. Fried, T.C. Killian, L. Willmann, D. Landhuis, S.C. Moss, D. Kleppner, and T.J. Greytak, Phys. Rev. Lett. 81, 3811 (1998).
[8] A. Setty, Ph.D. thesis, University of Minnesota, 1998 contains preliminary results.

[9] G. Baym and C. Pethick, Phys. Rev. Lett. 76, 6 (1996).

[10] M. Edwards, P.A. Ruprecht, K. Burnett, R.J. Dodd, and C.W. Clark, Phys. Rev. Lett. 77, 1671 (1996).

[11] K.G. Singh and D.S. Rokhsar, Phys. Rev. Lett. 77, 1667 (1996).

[12] F. Dalfovo, S. Giorgini, L.P. Pitaevskii, and S. Stingari, Rev. Mod. Phys. 71, 463 (1999).

[13] A.L. Fetter and J.D. Walecka, Quantum Theory of ManyParticle Systems (McGraw-Hill, San Francisco, 1971).

[14] E. Fermi, Riv. Sci. 7, 13 (1936).

[15] D.S. Jin et al., Phys. Rev. Lett. 77, 2360 (1996).

[16] M.-O. Mewes et al., Phys. Rev. Lett. 77, 988 (1996).

[17] M.R. Andrews, C.G. Townsend, H.J. Miesner, D.S. Purfee, D.M. Kurn, and W. Ketterle, Science 275, 637 (1997) 